\title{
Oligolectisme de Bombus brodmannicus delmasi TKALCŮ 1973 (Hymenoptera, Apidae) : observations et analyses
}

\author{
Par Simon Dellicour *, Roland De Jonghe **, Dorothée Roelants *** et Denis Michez ***
}

\begin{abstract}
Résumé. La spécialisation alimentaire pour le pollen est assez commune chez les abeilles solitaires (i.e. oligolectisme). Elle est par contre très rare chez les espèces sociales car les ouvrières doivent exploiter une grande variété de ressources pour suppléer aux besoins de la colonie tout au cours de sa relativement longue phénologie. Chez les bourdons, seulement trois cas d'oligolectisme sont recensés dans la littérature, Bombus gerstaeckeri MORAWITZ 1882, B. consobrinus DAHLBOM 1832 et B. brodmannicus VoGT 1909. Malgré la particularité de ce comportement, peu de données de terrain et palynologiques étayent l'hypothèse d'une spécialisation alimentaire chez ces trois espèces et ce, plus particulièrement pour B. brodmannicus. Cet article présente des observations de terrain ainsi que les résultats des analyses des pelotes de pollen récupérées sur des ouvrières de $B$. brodmannicus delmasi. Ces spécimens on été échantillonnés en mai 2011 dans les Alpes Occidentales dans le cadre d'une étude phylogéographique comparative visant à étudier l'impact de la spécialisation alimentaire chez plusieurs espèces d'abeilles.
\end{abstract}

Abstract. This article presents the field observations and the analyses of the pollen loads collected on Bombus brodmannicus delmasi TKALCU 1973 queens. Specimens were sampled in May 2011 in the framework of a comparative phylogeographic analysis which aims to study the impact of floral specialisation on several wild bee species.

Mots-clés. Apidae, Bombus brodmannicus, oligolectisme, Alpes.

\section{Introduction}

Les comportements de récolte de pollen sont très variés chez les abeilles. Les visites florales ne se font pas du tout de façon aléatoire mais témoignent bien d'un choix qui peut être plus ou moins étroit dans le temps et vis-à-vis de taxons floraux (Dötterl \& Vereecken 2010). La préférence alimentaire d'une abeille vis-à-vis d'un taxon de plante particulier (espèce, genre, famille) peut être caractéristique d'une espèce dans la globalité de sa distribution et demeurer inchangée de génération en génération. On appelle cette spécialisation alimentaire oligolectisme. Par exemple l'espèce Dasypoda hirtipes (FABRICIUS 1793) (Melittidae) est oligolectique sur les plantes de la famille des Asteraceae. Cela signifie que toutes les femelles de cette espèce, récoltent partout (dans ce cas ci de l'Angleterre à la Sibérie) et tout le temps leur pollen sur des plantes de la famille des Asteraceae comme Hypochoeris radicata L. 1753 ou Crepis foetida L. 1753 (Michez et al. 2008).

De nombreuses abeilles sont oligolectiques. Michener (2007) estime que plus de la moitié des espèces d'abeilles seraient spécialisées d'un point de vue alimentaire. Cependant, ce comportement n'est pas également réparti au sein des différentes familles et genres d'abeilles à l'échelle mondiale. Les espèces

* Evolutionary Biology \& Ecology, Université Libre de Bruxelles, CP 160/12, Av. F.D. Roosevelt 50, B-1050 Bruxelles, Belgique. E-mail: simon.dellicour@ulb.ac.be

** E-mail: roland.de.jonghe@telenet.be.

*** Laboratoire de Zoologie, Université de Mons, Place du Parc 20,

B-7000 Mons, Belgique. E-mail: denis.michez@umons.ac.be. solitaires sont généralement plus spécialisées dans leur régime alimentaire car leur phénologie est relativement courte par rapport aux espèces sociales (ex. : Apis, Bombus), ce qui leur permet de boucler leur cycle de vie pendant la floraison d'une seule ressource particulière.

Au sein du genre Bombus (Hymenoptera, Apidae), seules trois espèces sont actuellement considérées comme oligolectiques, au moins sur une partie de leur distribution. Premièrement, B. gerstaeckeri MORAWITZ 1882, est connu pour être spécialiste sur le genre Aconitum (Ranunculaceae) sur l'ensemble de sa distribution limitée à trois massifs montagneux européens les Pyrénées, les Alpes et les Carpates (Pittioni 1937; Delmas 1976; Rasmont 1988; Mahé 2008; Dellicour et al. 2012). Ensuite, les populations occidentales d'une autre espèce de Megabombus à distribution euro-sibérienne, B. consobrinus DAHLBOM 1832, sont aussi décrites comme oligolectiques sur le genre Aconitum (Löken 1961, 1973; D. Michez \& T. De Meulemeester, comm. pers.). Enfin, au sein des Pyrobombus, la sous-espèce $B$. brodmannicus delmasi TKALCU 1973, localisée dans certaines vallées des Alpes Occidentales (Figure 1), est considérée comme spécialiste (Delmas 1962, Rasmont 1988), alors que la sous-espèce B. brodmannicus brodmannicus VOGT 1909, localisée dans les montagnes du Caucase, est généraliste (Rasmont et al. 2009). Ce caractère oligolectique de la sous-espèce delmasi a été déduit des observations de terrain rapportant une préférence marquée de la sousespèce pour Cerinthe minor L. et Cerinthe glabra MILLER (Boraginaceae) (Delmas 1962; Tkalcu 1973; Delmas 1976; Rasmont 1988). Cependant, B. brodmannicus delmasi visite également d'autres genres comme par 

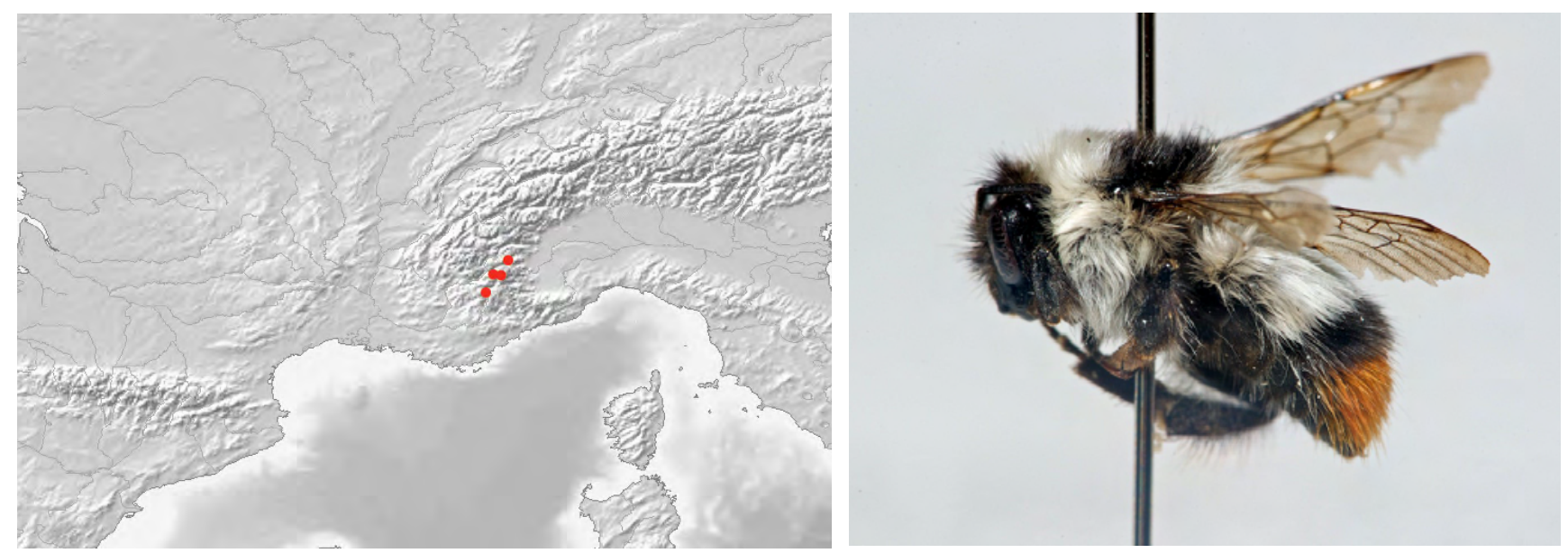

Figure 1. Carte d'échantillonnage des reines de la sous-espèce Bombus brodmannicus delmasi TKALCŮ dans les Alpes en Europe occidentale lors de l'expédition de mai 2011 (Photo NJ Vereecken).

exemple Calamintha, Epilobium, Scabiosa, Echium (Delmas 1962), Nepeta, Knautia (Delmas 1976) ou encore Stachys (Mahé 2008). Ce comportement à tendance généraliste concernerait plus particulièrement les mâles dont la phénologie est en partie décalée par rapport à la floraison des Cerinthe (Delmas 1976; Mahé 2008). Il s'agirait donc de visites pour la collecte de nectar.

Dans cet article, en plus de nos nouvelles observations de terrain, nous tenterons d'apporter une preuve plus formelle de ce caractère spécialiste via l'analyse palynologique réalisée à partir des pelotes de pollen récoltées sur les individus capturés. Nous présentons ensuite une description succincte de la technique utilisée pour conserver des reines de l'espèce vivantes en vue d'une mise en élevage.

\section{Sites d'échantillonnage et dernières observations de terrain}

Nous avons observé des reines de B. brodmannicus delmasi sur six sites différents (Figure 1, Tableau 1). A part sur le premier site où les reines étaient abondantes, seules une ou deux reines ont été capturées par site afin d'éviter de nuire aux populations existantes. L'ensemble des observations et captures se sont faites sur Cerinthe minor (Figure 2A). Ces plantes ont été trouvées, typiquement sur des versants Sud, entre les lacets d'une route menant vers un col (Figure 2B). Cependant, $B$. brodmannicus n'a été observé que sur les sites répertoriés dans le Tableau 1.

Malgré leurs passages très furtifs sur les fleurs de $C$. minor, les reines de $B$. brodmannicus étaient assez facilement identifiables grâce à leur pelage blanc neige typique de beaucoup de bourdons du Caucase (Figure 1; Delmas 1962; Tkalců 1973). Sur certains sites, la patience était de mise avant d'apercevoir la visite éclaire du bourdon sur sa plante favorite. Parmi les visiteurs de la Cerinthe minor à la même période et sur les sites d'observation de B. brodmannicus, nous avons identifié quelques reines de B. lapidarius (L. 1758), plusieurs reines et quelques ouvrières de $B$. pratorum (L. 1761) ainsi que plusieurs reines de B. sylvarum (L. 1761). Ces espèces collectaient également du pollen sur Cerinthe minor. A l'inverse de B. brodmannicus exclusivement
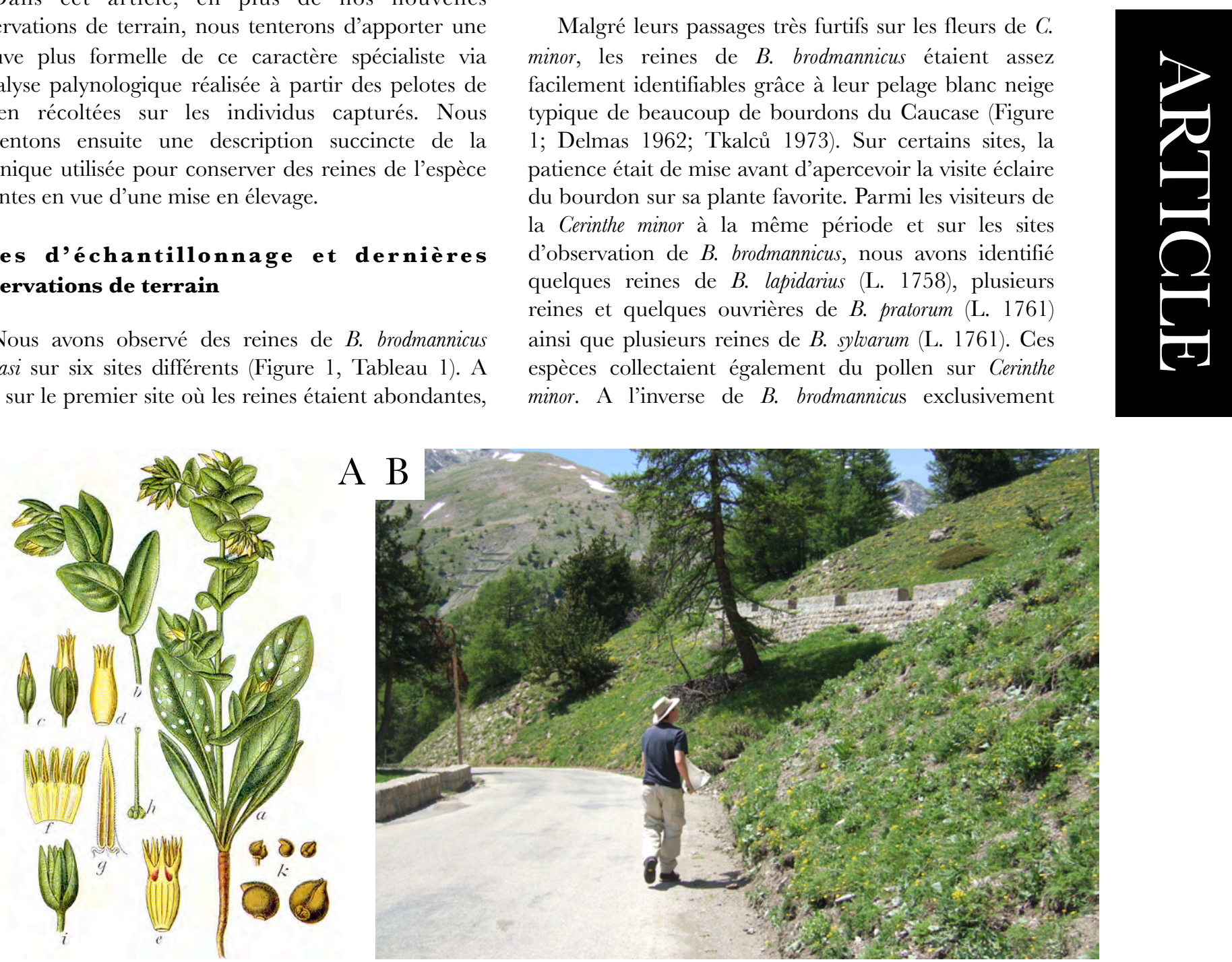

Figure 2. Prospections de sites à Cerinthe minor L. (Boraginaceae) dans les Alpes françaises. A. Détails d'une plante et des fleurs de C. minor (illustration J.G. Sturmfrom, Flore d'Allemagne lllustrée sur http://www.biolib.de); B. Exemple d'un biotope typique où est l'on trouve la Cerinthe minor dans les Alpes Occidentales. II s'agit de la photo d'un des lacets de la route qui monte vers le Col de la Cayolle (Photo B. Dellicour) 
Tableau 1. Détail des sites d'échantillonnage et d'observations de la sous-espèce Bombus brodmannicus delmasi TKALcŮ dans les Alpes françaises.

\begin{tabular}{|c|c|c|c|}
\hline Lieu-dit & Lat/Long $(\mathbf{W G S 8 4 )}$ & Récolte & Date, heure \\
\hline Sainte-Catherine (F) & $44.601944^{\circ} \mathrm{N}, 6.698889^{\circ} \mathrm{E}$ & 4 reines, 1 ouvrière & $28 . v .11,14 \mathrm{~h}$ \\
\hline Col de Vars (F) & $44.530278^{\circ} \mathrm{N}, 6.714722^{\circ} \mathrm{E}$ & 1 reine & $28 . v .11,17 \mathrm{~h}$ \\
\hline La Barge, Saint-Paul sur Ubaye (F) & $44.586944^{\circ} \mathrm{N}, 6.828000^{\circ} \mathrm{E}$ & 2 reines & $28 . v .11,19 \mathrm{~h}$ \\
\hline Col d'Allos (F) & $44.300000^{\circ} \mathrm{N}, 6.575833^{\circ} \mathrm{E}$ & 2 reines & $29 . v .11,09 \mathrm{~h} 30$ \\
\hline Col de la Cayolle (F) & $44.351667^{\circ} \mathrm{N}, 6.750556^{\circ} \mathrm{E}$ & - & $29 . v .11,14 \mathrm{~h} 00$ \\
\hline Le Roux (F) & $44.837778^{\circ} \mathrm{N}, 6.945000^{\circ} \mathrm{E}$ & 2 reines & $29 . v .11,19 \mathrm{~h} 00$ \\
\hline
\end{tabular}

observé sur la Cerinthe, ces espèces étaient tout aussi régulièrement observées sur d'autres espèces de plantes localisés entre les Cerinthe minor comme par exemple Trifolium pratense (L. 1753).

\section{Analyse qualitative des pelotes de pollen}

L'analyse palynologique a été effectuée sur des pelotes de pollen prélevées sur 8 des 12 individus capturés sur le terrain. Cette étude de la composition en pollens a été réalisée par acétolyse selon le protocole de Westrich \& Schmidt (1986). Les pelotes ont été délicatement prélevées des corbeilles et placées individuellement dans des tubes de type Eppendorf. Ensuite, le pollen a été déshydraté dans de l'acide acétique glacial puis a subi une acétolyse dans une solution d'anhydride acétique et d'acide sulfurique concentré (9:1). Le pollen acétolysé a ensuite été rincé plusieurs fois dans une solution d'eau et d'éthanol 96\% (3:1). Les échantillons ont été montés sur lame à l'aide de gélatine.

Les pollens de Cerinthe sp. ont été comptés sous microscope au grossissement x200 selon le protocole de Müller (1996) et le pourcentage de présence en nombre a été calculé. Le degré de charge des pelotes a été au préalable estimé selon une échelle allant de 1 à 5 . Le niveau 1 correspond à un remplissage allant de 0 à $20 \%$ et le niveau 5 à une charge complète de $100 \%$.

Tableau 2. Résultats de l'analyse qualitative des pelotes de pollen prélevées sur 8 individus de Bombus brodmannicus delmasi TKALCU

\begin{tabular}{|c|c|c|c|}
\hline Individu & Origine & $\begin{array}{c}\text { Degré de } \\
\text { charge en } \\
\text { pollen }\end{array}$ & $\begin{array}{c}\text { \% de pollen } \\
\text { de Cerinthe } \\
\text { sp. }\end{array}$ \\
\hline 1 & Sainte-Catherine & 1 & 100 \\
\hline 2 & Sainte-Catherine & 1 & 100 \\
\hline 3 & Col d'Allos & 1 & $>99$ \\
\hline 4 & Col d'Allos & 1 & 100 \\
\hline 5 & Le Roux & 4 & $>99$ \\
\hline 6 & Le Roux & 4 & 100 \\
\hline 7 & La Barge & 4 & 100 \\
\hline 8 & Le Barge & 1 & $>99$ \\
\hline
\end{tabular}

Comme l'indique le résultat des analyses (Tableau 1), toutes les pelotes des spécimens étudiés présentent un contenu en pollen de Cerinthe sp. > à 99\%, quelle que soit leur provenance. Néanmoins, ces résultats doivent être pris avec précaution étant donné le nombre d'échantillons investigués et le degré de charge en pollen faible d'une partie des pelotes étudiées. Ces analyses, couplées aux observations de terrain et aux observations reprises dans la littérature (Delmas 1962; Rasmont 1988), tendent très clairement à confirmer le caractère oligolectique de la sous-espèce $B$. brodmannicus delmasi. Il est toute fois également précisé que cet oligolectisme est observé sur le temps de la phénologie des Cerinthe. En effet, une fois celle-ci terminée, $B$. brodmannicus delmasi, et plus particulièrement les mâles dont la phénologie est plus tardive, se retrouve également sur d'autres plantes-hôtes (Delmas 1976; Mahé 2008).

\section{Transport et élevage des reines}

A côté de l'analyse du caractère oligolectique de $B$. brodmannicus delmasi, les objectifs de l'expédition étaient de récolter du matériel frais pour en extraire l'ADN et réaliser une étude phylogéographique de cette sousespèce en comparaison avec la sous-espèce généraliste du Caucase (B. brodmannicus brodmannicus) mais aussi avec son espèce sœur ( $B$. pratorum), toutes deux déjà échantillonnées sur l'ensemble de leur aire de distribution. Le deuxième objectif était de réaliser l'élevage de plusieurs reines dans le but d'effectuer une étude éthologique de cette sous-espèce particulière. Nous présentons ici la technique utilisée pour capturer et maintenir ces reines en vie entre le lieu de capture et la salle d'élevage. Cette technique peut être appliquée facilement aux autres espèces de bourdons.

Sur les sites de récolte, les spécimens ont été attrapés au filet et ensuite introduits dans des boites d'allumettes fermées à l'aide d'élastiques. De retour au véhicule, chaque boîte d'allumette était ouverte face à l'ouverture d'une petite cellule individuelle (Figure 3). En raison de la luminosité extérieure possible grâce à la fine grille des cages, les spécimens capturés quittaient assez vite leur boîte pour pénétrer dans leur petite cellule. Leur nutrition était assurée par de l'ouate imbibée d'une solution stérilisée d'eau sucrée. Cette ouate imbibée 

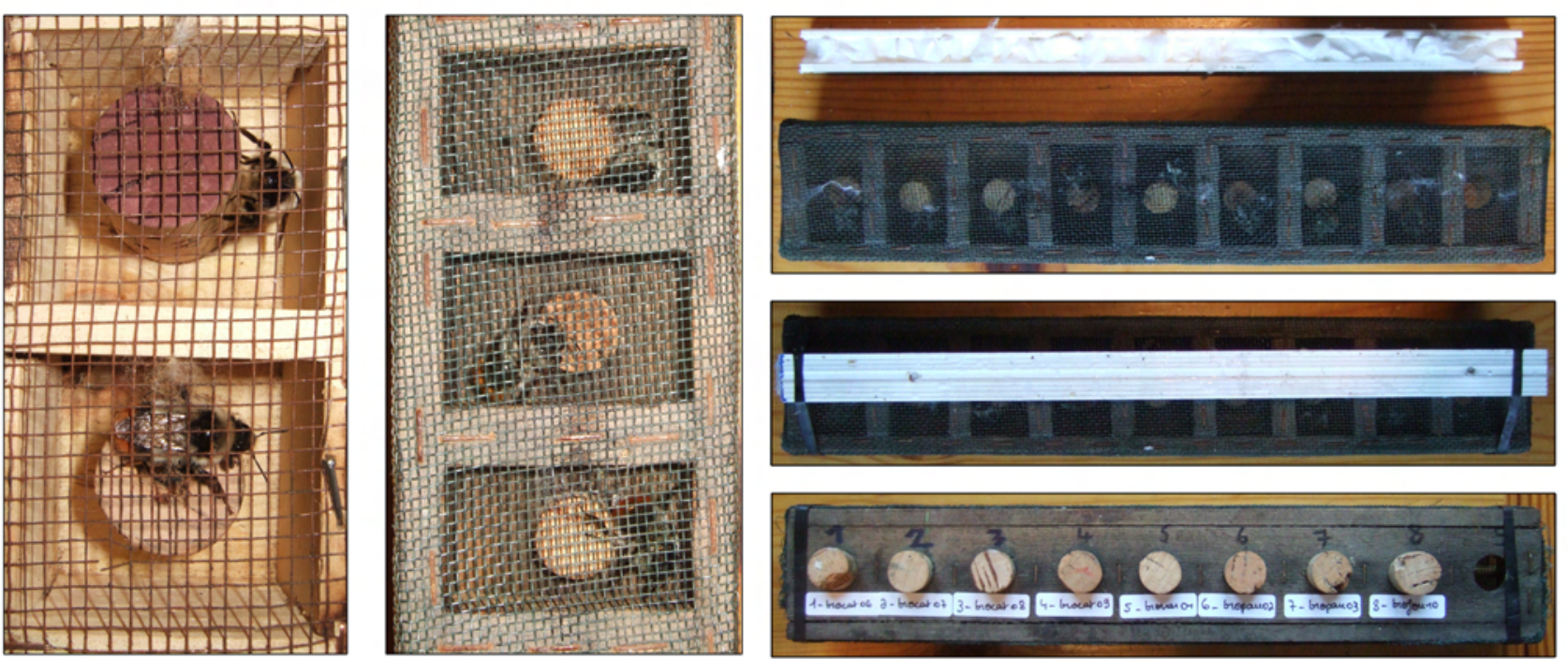

Figure 3. Cellules individuelles dans lesquelles ont été maintenues en vie les reines de Bombus brodmannicus delmasi Tkalců jusqu'à leur retour en Belgique (gauche) et dispositif installé pour nourrir les reines : de l'ouate imbibée d'eau sucrée maintenue contre les cellules à l'aide d'élastiques (droite). Photos S. Dellicour

était maintenue contre la grille des cages à l'aide d'un support fixé aux cellules à l'aide de deux élastiques (Figure 3). Lors des trajets en voiture, les petites cages étaient maintenues dans un bac frigo dont l'inertie thermique était assurée à l'aide de bouteilles d'eau gelées. En attente de leur retour en Belgique cinq jours plus tard, les reines ont été maintenues au frigo à $3{ }^{\circ} \mathrm{C}$ afin de provoquer une torpeur artificielle. Les cages ont été sorties 15 minutes à température ambiante tous les jours le temps de remplacer l'ouate par de la nouvelle ouate fraîchement imbibée par la solution sucrée. De retour en Belgique, ces reines ont été placées en élevage (Figure 3) dans le but d'obtenir des informations éthologiques et de compléter l'étude de leur caractère spécialiste.

\section{Remerciements}

Nous tenons tout d'abord à remercier le FNRS qui finance le projet de recherche global sur l'étude de la spécialisation alimentaire chez les abeilles (FRFG 2.4613.10). Merci à B. Dellicour et S. Bellens pour l'immense et indispensable aide sur le terrain, et à $\mathrm{T}$. Lecocq pour sa précieuse aide pour le repérage des sites à Cerinthe lors de l'été 2010. Merci aussi finalement à G. Mahé et P. Rasmont pour leurs informations sur les sites d'échantillonnage.

\section{Références bibliographiques}

Delmas R, 1962. Notes zoogéographiques et systématiques sur les Bombidae. I. - Le Bombus brodmannicus VOGT des Alpes françaises. Annales de l'Abeille 5(3): 175-179.

Delmas R, 1976. Contribution à l'étude de la faune française des Bombidae (Hymenoptera, Apoidea, Bombidae). Annales de la Société Entomologique de France (n.s.) 12: 247-290.
Dötterl S \& Vereecken NJ, 2010. The chemical ecology and evolution of bee flower interactions: a review and perspectives. Canadian Fournal of Zoology 88: 668-697.

Løken A, 1961. Bombus consobrinus DAHLBOM, an oligolectic bumblebee (Hymenoptera, Apidae). Proceeding of the XIth Int. Congr. Ent. 1960 1: 598-603.

Løken A, 1973. Studies on Scandinavian Bumblebees (Hymenoptera, Apidae). Norsk Entomologisk Tidsskrift 20: 1-218.

Mahé G, 2008. Bourdons rares du Parc Naturel Régional du Queyras (Hautes-Alpes, France). OSMIA 2: 21-25.

Michez D, Patiny S, Rasmont P, Timmermann K \& Vereecken NJ, 2008. Phylogeny and host-plant evolution in Melittidae s.l. (Hymenoptera: Apoidea). Apidologie 39: 146-162.

Müller A, 1996. Host-plant specialization in Western Palearctic Anthidiine bees (Hymenoptera: Apoidea: Megachilidae). Ecological Monographs 66: 235-257.

Pittioni B, 1937. Bestäubung und Nektarraub beim Gelben Eisenhut (Aconitum vulparia RCHB). Aus der Heimat, Stuttgart 50: 209-213

Rasmont P, 1988. Monographie écologique et zoogéographique des bourdons de France et de Belgique (Hymenoptera, Apidae, Bombinae). T h è s e d e doctorat, Faculté des Sciences agronomiques de l'Etat, Gembloux, 309 p.

Rasmont P, Aytekin AM, Kaftanoglu O \& Flagothier D, 2009. The bumblebees of Turkey. Atlas Hymenoptera, Université de Mons, Gembloux Agro-Biotech, Mons, Gembloux, http://www.zoologie.umh.ac.be/hymenoptera/page.asp?ID=103

Özbek H, 1998. On the bumblebee fauna of Turkey: II. The genus Pyrobombus (Hymenoptera, Apidae, Bombinae). Zoology In The Middle East 16: 89-106.

Robertson C, 1925. Heterotrophic bees. Ecology 6: 412-436.

Tkalců B, 1973. Taxonomie von Pyrobombus brodmannicus (VOGT) (Hymenoptera, Apoidea, Bombinae). Acta entomologica Bohemoslovaca 70(4): 259-268.

Westrich P \& Schmidt K, 1986. Methoden und Anwendungsgebiete der Pollenanalyse bei Wildbienen (Hymenoptera, Apoidea). Linzer biologische Beiträge 18: 341-360. 